\title{
The Second Palomar-Leiden Trojan Survey
}

\author{
C. J. Van Houten, I. Van Houten-Groeneveld, and M. Wisse-Schouten \\ Leiden Observatory, P.O. Box 9513,2300 RA Leiden, The Netherlands \\ C. Bardwell, ANd D. W. E. Green \\ Smithsonian Astrophysical Observatory, 60 Garden Street, Cambridge, Massachusetts 02138
}

AND

\section{T. GeHRELS}

Lunar and Planetary Laboratory, University of Arizona, Tucson, Arizona 85721

Received April 16, 1990; revised February 1, 1991

The 1973 Trojan survey of a region close to the preceding Lagrangian point is discussed in this paper. This region consists of four overlapping fields and one field for the photometric calibration, centered on Selected Area 68. Orbital elements of most of the 1504 asteroids found have been published in the Minor Planet Circulars. Among these are 5 Hungaria-type asteroids, 12 of the Hilda type and 55 Trojans. The accuracy of the orbits is somewhat lower than in the Palomar-Leiden survey of 1960 , because of the shorter arc in the present investigation. A discussion of the degree of completeness of this material shows that the (photographic) magnitude interval $19.5-20.0$ is only $50 \%$ complete; to obtain completeness down to magnitude 20.0 the number of found Trojans brighter than this limit has to be increased by 9 . In combination with the surveys of 1960 and 1965 an improved distribution of the number of Trojans as a function of distance to the preceding Lagrangian point (L4) has been constructed. A preliminary discussion, including the result on L5, yields a value 2.0 for the ratio of Trojans around L4 and L5, along the ecliptic. The L5 Trojans appear to be deficient in orbits of small inclination. Taking this into account we find this ratio again to be $\mathbf{2 . 0}$ for the total Trojan clouds around L4 and L5. Possibly, however, high-inclination Trojans were systematically overlooked in this survey. If this would be true, the ratio $N(\mathrm{~L} 4) / N(\mathrm{L5})$ is increased to 2.3 . The uncertainty of these numbers can be estimated to be $\mathbf{\pm 0 . 5}$. 1991 Academic Press, Inc.

\section{INTRODUCTION}

Trojan asteroids, which move in or near the Jupiter orbit, consist of two groups, around two Lagrangian points of the Sun-Jupiter system. The point preceding Jupiter in its orbit is denoted by $\mathrm{L} 4$, and the point following Jupiter by $\mathrm{L} 5$. The problem of whether the numbers of asteroids around these two points, down to a fixed magnitude limit, are equal had not been extensively investigated until recently. To solve this problem we set up three Trojan surveys, in 1971 (L5), 1973 (L4), and 1977 (L5). We refer to the surveys as T-1, T-2, and T-3. Together with the quick survey of 1965 (L4) (Van Houten $e t$ al. 1970a; the Lagrangian point investigated there was erroneously denoted as $\mathrm{L} 5$ in that paper) and the Palomar-Leiden survey of 1960 (PLS; Van Houten et al. 1970b), also for L4, this should yield sufficient material to answer this question.

The surveys mentioned above cover only part of the regions in which Trojans are found; in view of the large inclination of several Trojan orbits a complete coverage to the limiting magnitude of the PLS is practically impossible. The fields of the present surveys are all centered on the ecliptic. If the orbital elements of the Trojans are known and the numbers are sufficiently large for a statistical treatment, the density of Trojans outside the ecliptic zone can be computed, so that in principle a smaller area coverage, provided it contains the ecliptic region, is sufficient to derive densities for the complete surroundings of a Lagrangian point.

\section{MATERIAL AND REDUCTION}

The 1973 survey consists of four fields with 15 plates for each field, and 16 for the photometry calibration on a standard sequence in Selected Area 68; in all 76 plates. They were taken with the Oschin $122-\mathrm{cm}$ Schmidt telescope of Palomar Observatory by Gehrels; the dates of exposure were 1973 September 19, 20, 24, 25, 29, 30, 
TABLE I

Plate Centers of the Fields Photographed in this Survey

\begin{tabular}{|c|c|c|c|c|c|c|c|}
\hline \multirow{2}{*}{$\frac{\text { No. }}{1}$} & \multicolumn{3}{|c|}{ RA (1950) } & \multicolumn{3}{|c|}{ Declination (1950) } & \multirow{2}{*}{$\frac{\text { Distance from } \mathbf{L}}{-9^{\circ} .2}$} \\
\hline & $0^{h}$ & $8^{m}$ & $5^{\mathrm{s}}$ & $+2^{\circ}$ & $8^{\prime}$ & $0^{\prime \prime}$ & \\
\hline 2 & 0 & 32 & 23 & +4 & 14 & 13 & -4.2 \\
\hline 3 & 0 & 8 & 35 & -3 & 28 & 36 & -10.9 \\
\hline 4 & 0 & 30 & 32 & -1 & 1 & 29 & -6.0 \\
\hline 5 & 0 & 13 & 29 & +14 & 32 & 0 & \\
\hline
\end{tabular}

October 4, 5 UT. Except for September 20 each field was photographed twice on these nights, with a time interval of about $90 \mathrm{~min}$; the exposure time was $12 \mathrm{~min}$. The plates were guided on mean Trojan motion. The plate centers of the four program fields ( 1 to 4 ) and the Selected Area field (5) are listed in Table I. For the program field these centers are only valid for the four central dates: for the other dates they were shifted in accordance with the mean Trojan motion.

The plates were blinked with the blink microscope of the Lunar and Planetary Laboratory of the University of Arizona; this instrument is on loan to the Leiden Observatory. One blink pair of each program field was blinked by Van Houten; as a check Van Houten-Groeneveld blinked a different plate pair of fields 1 and 4 each. Moreover, Van Houten-Groeneveld blinked one pair of plates of field 5 . For the latter three plate pairs the blink comparator of the Catholic University at Nijmegen was used. Positions of the Trojans and other slowly moving asteroids found by Van Houten were measured by him at the Kapteyn Laboratory at Groningen. Orbital elements based on these positions were computed by Paul Herget of Cincinnati Observatory and were published in Minor Planet Circulars (MPC) 4285-4294; they received the preliminary designations $1973 \mathrm{SL}$ to $1973 \mathrm{SC} 2$. All other asteroids found in this survey, except those identical with numbered asteroids and part of those identical with preliminarily designated asteroids, received a running number followed by the symbol T-2. The first digit of the running number refers to the field in which the asteroid was found, in accordance with the numbering in Table $I$.

Photographic magnitudes of the asteroids found were measured by M. Wisse-Schouten with the Sartorius Iris photometer of the Leiden Observatory. Standard magnitudes were obtained from the exposures on Selected Area 68 taken in the same night as the asteroid field exposures. The magnitudes used were those published by Stebbins et al. (1950) and, for $m>18.5$, they have been determined by Baum (unpublished) and were kindly forwarded to us. The magnitudes were corrected for airmass differences between program fields and Selected Area, adopting an extinction coefficient of 0.4 mag per unit airmass. The magnitudes were also corrected for trail effects with the relation derived in the PLS; the trail corrections are taken to be proportional to the lengths of the trail.

The plates were measured for positions by Van HoutenGroeneveld with the blink comparator of the Catholic University at Nijmegen. This instrument is provided with electronic scales for position measurements, each allowing a precision of $\pm 0.012 \mathrm{~mm}$, which corresponds to 0.8 on our plates. It has the advantage that the two blink plates can be measured simultaneously with respect to one system of reference stars. Orbital elements based on these positions were computed by Green and Bardwell of the Smithsonian Astrophysical Observatory (Cambridge, MA). In this process the objects 1973 SL to 1973 SC2 were remeasured and new orbital elements were computed from these positions only. The T-2 orbital elements have been published in Minor Planet Circulars 14904-14930, except for those asteroids for which an orbit was already known. All further reductions based on these data were made by Van Houten.

\section{PRECISION OF POSITIONS AND ORBITAL ELEMENTS}

The internal precision of the measured positions was derived from the residuals with respect to positions computed from 39 well-determined orbits, distributed uniformly in magnitude. The average difference between measured and computed position, without regard to sign, is 0.63 and 0.74 for the $x$ and $y$ coordinate, respectively, yielding an internal error of 0.97 per position, nearly equal to the value found in the T-3 survey (Van Houten-Groeneveld et al. 1989). No correlation of accuracy with magnitude was apparent.

The precision of the orbital elements was determined from the numbered asteroids by comparing their elements from the present material with those published in the "Minor Planet Ephemerides" (Institute for Theoretical Astronomy, Leningrad) for 1989 and from the Minor Planet Circulars for asteroids numbered later. For this comparison 28 orbits were available based on a 16-day arc in the present material, and 6 orbits based on a 6-day arc, for objects which ran out of the area photographed, or entered it during the observational time interval. This accuracy is not only dependent on the arc length in time, but also on the arc length in degrees; slowly moving objects yielding less accurate orbital elements than objects with average motion. The Trojans were therefore excluded from this comparison, and are considered separately below.

The average differences without regard to sign are listed in Table II. It is seen that these residuals, for the $16^{\mathrm{d}} \mathrm{arc}$, are about twice as large as for the PLS quality-1 $(Q=1)$ values (these are the PLS orbits of the highest quality). This does not agree with the results of the T-3 survey, 
TABLE II

Average Differences without Regard to Sign between Orbital Elements of Numbered Asteroids from the 1989 Minor Planet Ephemerides and the Minor Planet Circulars (Trojans Excluded) and Those from 16-Day and 6-Day Arcs in the Present Material, Compared to PLS Quality 1 and Quality 3 Results

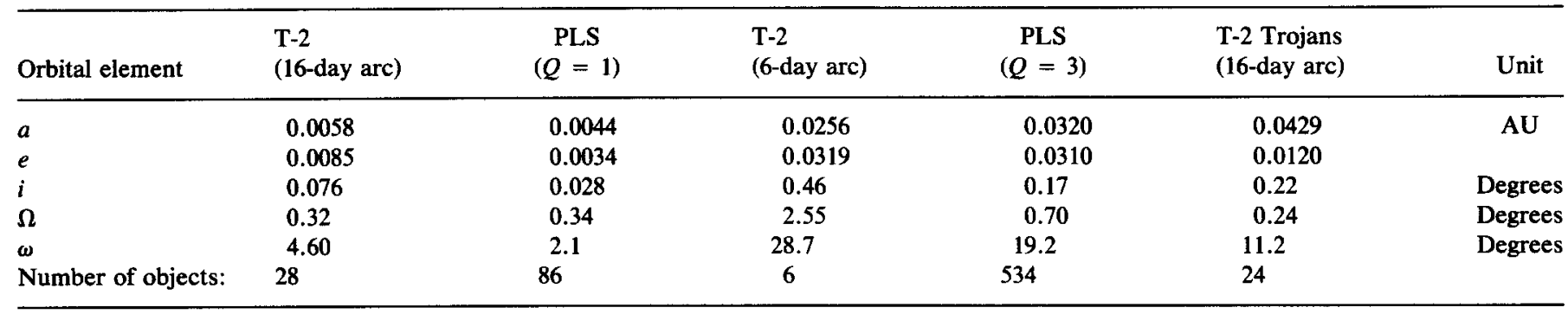

Note. For the derivation of the Trojan values, see text.

where it was found that their $15^{\mathrm{d}}$ arc orbits were of about the same accuracy as the $Q=1$ orbits of the PLS. The explanation must be sought in the fact that the T-3 objects were generally found farther from the ecliptic than the T-2 objects and that therefore their orbital curvature was more clearly visible.

The six orbits based on a 6-day arc suggest that their accuracy is about equal to that of the quality-3 orbits of the PLS, which are based on a 9-day arc. The material available for this comparison is too small to say more.

A method to derive the accuracy of the Trojan orbits is the following: the measurements of the Trojan positions by Van Houten and the orbital elements derived from them by Herget are independent of the positional measurements by Van Houten-Groeneveld and the orbital elements computed from them by Bardwell and Green. Differences between the two sets of orbital elements should only be caused by errors of measurement in the two sets of positions. We assume that the two sets of elements are of the same precision, and thus the average differences found between them were divided by $\sqrt{2}$ to obtain the accuracy of a single set. They are collected in Table II under the heading "T-2 Trojans." The accuracy found is approximately similar to that of PLS $Q=3$ orbits.

\section{QUALITY OF PHOTOMETRY}

The magnitudes derived here can only be compared with those from other programs by computing the absolute magnitude, for which distance to Sun and Earth, and the phase angle are reduced to standard values. For this comparison we used the same definition of absolute magnitude as that in the PLS, where a phase function derived from the PLS material itself is used. The linear part of this phase function has a slope of 0.039 mag per degree and is extrapolated to phase zero to derive the absolute magnitude. This quantity is denoted as $g$; it is a photographic magnitude. The opposition effect adopted is that found by
Gehrels (1956) for Massalia. The modern convention of defining the absolute magnitude is not followed here, in order to obtain an easy comparison with the PLS values. The modern definition is followed in the MPCs listing of the orbital elements of the T- 2 asteroids (see above).

The standard values of absolute magnitude which were used for comparison with the $g$ values derived from this program were taken from the TRIAD (Tucson Revised Index of Asteroid Data) file (Bowell et al. 1979), and the PLS. As a rule only those objects which have a reliable orbit in the present program were used. This yielded 16 objects from the TRIAD file and 15 from the PLS. They are listed in Table III, where the apparent magnitude in the present program is given together with the absolute magnitude derived and the standard value from the TRIAD file or the PLS. It is clear that a magnitude-dependent difference exists between the T- 2 absolute magnitudes and the standard values. Average values of this difference are shown in Fig. 1. We decided to correct the present magnitudes for this effect; the correction used is

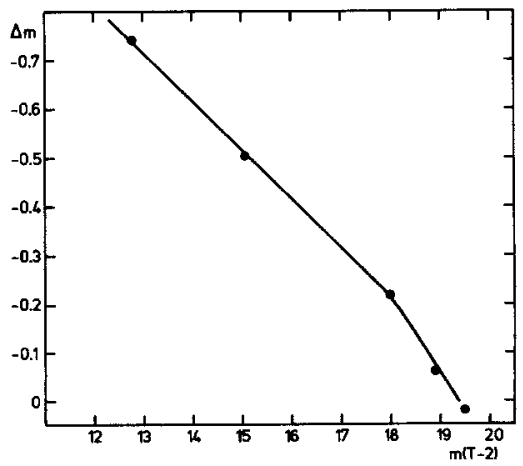

FIG. 1. Average differences between our photometry and standard values (T-2 minus standard) as a function of T-2 photographic apparent magnitude. The full-drawn line indicates the corrections actually applied. 
the full-drawn line in Fig. 1. The corrected T-2 $g$ values are also listed in Table III. Their average differences with the standard absolute magnitudes is $-0.02 \pm 0.08$ (m.e.) for the TRIAD values and $0.00 \pm 0.04$ (m.e.) for the PLS values. Apparently no systematic difference is left after the correction.

What caused this systematic effect? The form of the systematic deviation and its disappearance at about a magnitude above the plate limit seems to suggest an insufficient correction for trail effects. It turned out that trail corrections 2.6 times those actually applied gave a good agreement between observed and standard magnitudes. The average differences are $-0.05 \pm 0.08$ (m.e.) for the TRIAD values and $+0.01 \pm 0.05$ (m.e.) for the PLS values. This is of the same quality as the result obtained by applying the systematic error curve of Fig. 1. Since correction of the trail effect as indicated above did not give a better result compared to the use of Fig. 1, the latter was used throughout to eliminate the systematic errors of the photometry.

The 1973 plates were much more fogged than those of the PLS, presumably because of a longer developing time. It seems possible that this has influenced the trail effect, in the sense found above.

In this respect it must be remarked that no plates were taken in 1973 to calibrate the trail effect for the T-2 material. If this had been done, it would have settled this question. To take plates afterward seems rather ineffective, since it is probably impossible after all these years to duplicate all factors influencing the photometry of this program.

Although trail corrections 2.6 times those actually applied eliminate the systematic difference found above, it is by no means certain that this is the right explanation. The cause of this difference in magnitude is still unclear.

An indication that the magnitude corrections applied here are correct can be seen from Fig. 2. The $\log N(m)$ versus $m$ relation depicted here agrees with the PLS result, after the magnitude correction has been applied.

Five comparisons could be made between T-3 and T-2 absolute magnitudes; they are collected in Table IV. It is seen that a systematic error of the T-3 magnitude zero point found earlier (Van Houten-Groeneveld et al. 1989), $+0.19 \pm 0.05$ (m.e.), is found again here, but now diminished to $+0.09 \pm 0.05$ (m.e.). Within their margin of error the two determinations are in agreement.

\section{COMPLETENESS OF THE PRESENT SURVEY DOWN TO MAGNITUDE 20.0}

For the determination of the number of Trojans down to 20.0 mag the numbers counted must be corrected for objects missed during the blinking of the plates. The most

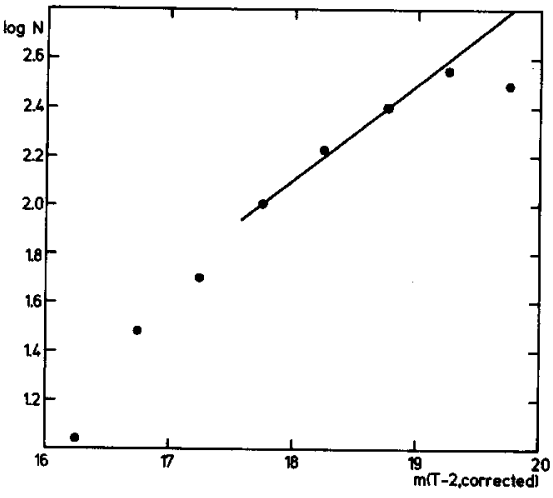

FIG. 2. The logarithms of the numbers of asteroids found in the fields 1 to 4, counted in half-magnitude intervals. The solid line has a slope of 0.39 per magnitude interval and is drawn by eye through the points in the interval 17.5-19.5.

direct way is by comparing the results of blinking two plate pairs of the same field; the ratio of the number of objects found twice to those found once immediately yields the degree of completeness. This method was not possible here, because for two of the fields only one plate pair was blinked, and the two plate pairs of the other two fields were blinked by two different people and with different instruments. The method chosen here is counting in half-magnitude intervals all asteroids found in the survey. The form of this magnitude-frequency relation is known from the PLS, down to magnitude 20.0. As found there, the logarithm of the numbers of asteroids counted in this way increases by 0.39 per magnitude interval with increasing magnitude, starting from magnitude 17.5. The numbers counted in the four program fields are shown in Table $\mathrm{V}$ and in Fig. 2, where the best fitting line with slope 0.39 per magnitude is drawn through the points. The line, when extrapolated to magnitude 20 , shows that the magnitude interval 19.5-20.0 is about 50\% incomplete: it should contain 617 asteroids, while only 306 were found. It is assumed here that the degree of completeness of the Trojans is equal to that of the field asteroids as found above. To apply a correction to the Trojan numbers based on this result is not so easy, because the distribution of the Trojans found here, as a function of magnitude, is rather irregular, as shown in Table VI. This is most probably caused by the relatively small number of T-2 Trojans found. Therefore it is better to average the Trojan numbers in full magnitude intervals. The degree of completeness of the asteroids found in the magnitude interval 19.0-20.0 is 0.7 , as follows from Table V. In this interval 20 Trojans were found, so that $3 / 7 \times 20=9$ Trojans still have to be added to the program fields to make the material complete down to $20.0 \mathrm{mag}$. The asteroids found in the Selected Area field are not included in these statistics, 
TABLE III

Apparent and Absolute Magnitudes ( $g$ ) of T-2 Photometry Compared to Absolute Magnitudes of the TRIAD File (T) and Palomar-Leiden Survey $(P)$, and Their Differences before $\left(\Delta_{1}\right)$ and after $\left(\Delta_{2}\right)$ the Systematic Correction of the T-2 Photometry

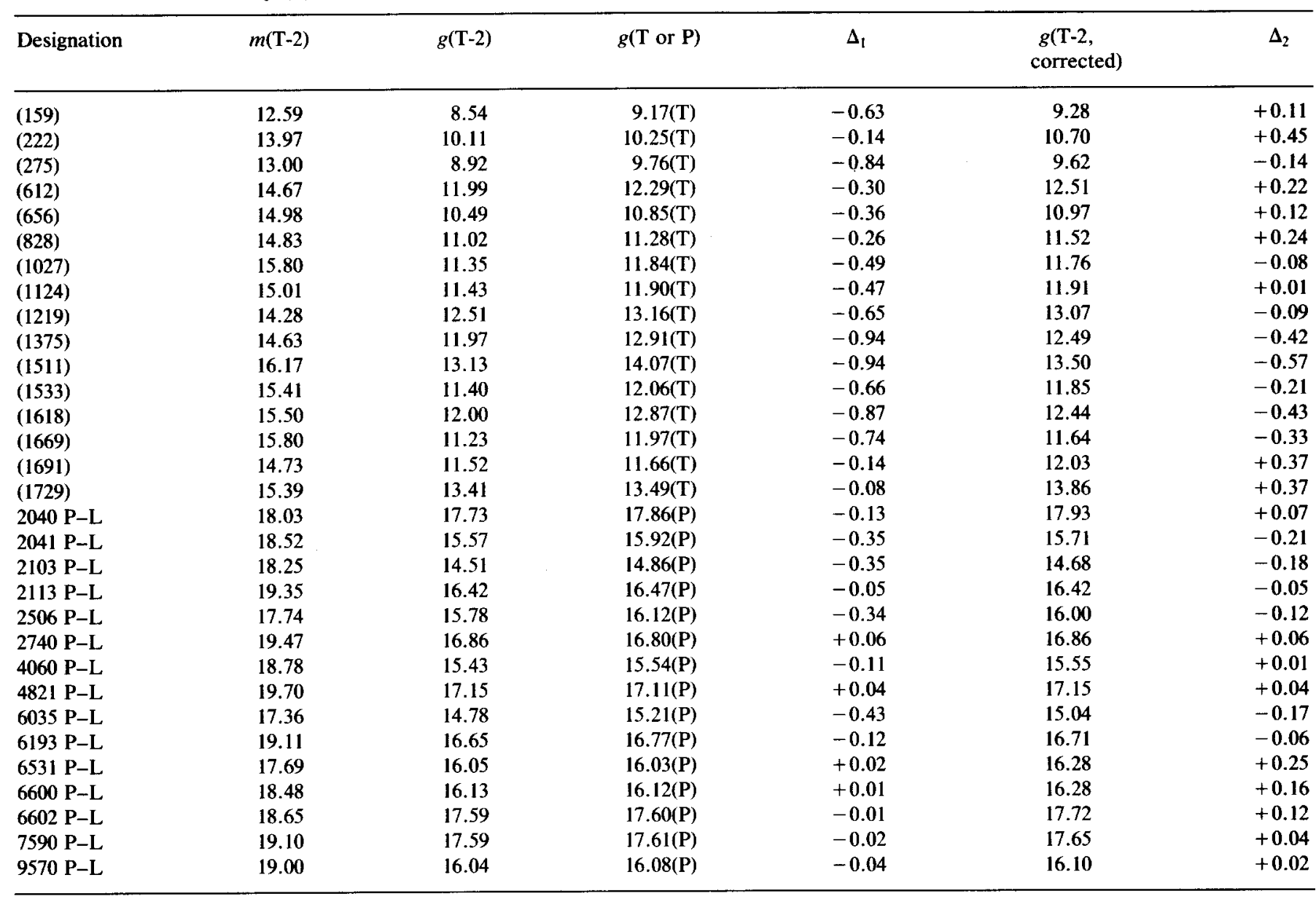

this field being outside the ecliptic region. Moreover, the number of Trojans found in this field, only nine, is too small for a statistical treatment.

The determination of the degree of completeness of the 1977 survey (Van Houten-Groeneveld et al. 1989) yielded the result that this material is complete down to photo-

TABLE IV

Absolute Magnitudes $g$ of the Corrected T-2 Photometry Compared with the Corresponding T-3 Values

\begin{tabular}{cccc}
\hline Designation & $g(\mathrm{~T}-2$, corrected $)$ & $g(\mathrm{~T}-3)$ & Difference \\
\hline $1060 \mathrm{~T}-3$ & 16.69 & 16.66 & +0.03 \\
$3180 \mathrm{~T}-3$ & 17.65 & 17.56 & +0.09 \\
$3217 \mathrm{~T}-3$ & 16.11 & 16.16 & -0.05 \\
3317 T-3 & 15.70 & 15.56 & +0.14 \\
$3731 \mathrm{~T}-3$ & 17.52 & 17.26 & +0.26 \\
\hline
\end{tabular}

graphic magnitude 20.0. In view of the small numbers involved we decided to reinvestigate the completeness of this material in the same way as for the 1973 material: by counting the asteroids found in half-magnitude intervals and comparing the relation between numbers and magnitude found in the PLS. This method is much more powerful, because much larger numbers are used. The result was that in the 1977 material the interval $19.5-20.0$ mag is only two-thirds complete. All numbers referring to T-3 used in the following discussion have been corrected for this incompleteness.

\section{ASTEROIDS FOUND}

The total number of asteroids found in this program, for which orbits were derived (part of them with assumed eccentricity) is 1505. Of these, 55 are Trojans, 5 are Hungaria-type objects, 12 are Hilda-type objects, and 3 
TABLE V

Numbers of Asteroids Counted in Half-Magnitude Intervals, $N(m)$, Found in the Fields 1 to 4

\begin{tabular}{ccc}
\hline Magnitude interval & $N(m)$ & $\log N(m)$ \\
\hline $16.0-16.5$ & 11 & 1.04 \\
$16.5-17.0$ & 30 & 1.48 \\
$17.0-17.5$ & 50 & 1.70 \\
$17.5-18.0$ & 100 & 2.00 \\
$18.0-18.5$ & 172 & 2.24 \\
$18.5-19.0$ & 254 & 2.40 \\
$19.0-19.5$ & 355 & 2.55 \\
$19.5-20.0$ & 306 & 2.49 \\
$20.0-20.5$ & 35 & 1.54 \\
\hline
\end{tabular}

(possibly 4) are Mars-crossers, two of which approached the Earth to within about $0.5 \mathrm{AU}$ during the observations. Of the orbits $70 \%$ are based on an arc length of 16 or 15 days, which means that also for faint asteroids orbits with reasonable accuracy could be obtained.

At the time this survey was photographed, the fields contained 17 definitely and 20 preliminarily numbered asteroids; at present these numbers have increased to 41 and 102, respectively. Identifications with PLS objects were 25 , and with T-3 objects 14 . No known Trojans were found in this survey at the time it was made; at present identifications with 4 definitely and 8 preliminarily numbered Trojans are known.

\section{DISTRIBUTION OF TROJANS ALONG THE ECLIPTIC AROUND L4}

With the conclusion of the T-2 program the density of L4 Trojans along the ecliptic can in principle be determined. The other surveys contributing to this function are the PLS and the quick survey of 1965 (Van Houten et al. 1970a). The two latter surveys were already used to construct such a relation (Van Houten et al. 1970a); this

TABLE VI

Number of Trojans Found in the T-2 Survey, Counted in HalfMagnitude Intervals

\begin{tabular}{ccc}
\hline & \multicolumn{2}{c}{ Number of Trojans found } \\
\cline { 2 - 3 } Magnitude interval & Field 1 to 4 & Field 5 \\
\hline $16.5-17.0$ & 1 & \\
$17.0-17.5$ & 2 & 1 \\
$17.5-18.0$ & 5 & \\
$18.0-18.5$ & 5 & 2 \\
$18.5-19.0$ & 8 & 4 \\
$19.0-19.5$ & 4 & 2 \\
$19.5-20.0$ & 16 & \\
$20.0-20.5$ & 5 & \\
\hline
\end{tabular}

TABLE VII

Numbers of Trojans Brighter than 20.0 mag in Opposition Found near L4 along the Ecliptic, in a Field 6.5 × 6.5 Wide, as a Function of the Heliocentric Angular Distance to L4 ( $\Psi$ )

\begin{tabular}{|c|c|c|}
\hline$\Psi$ & $\begin{array}{l}\text { Number of Trojans } \\
\text { brighter than } \\
m=20.0\end{array}$ & Source \\
\hline$-10^{\circ} 9$ & 12 & 1973 field 3 \\
\hline-9.2 & 11 & 1973 field 1 \\
\hline-6.0 & 14 & 1973 field 4 \\
\hline-4.2 & 14 & 1973 field 2 \\
\hline+4.5 & 16 & 1965 field A \\
\hline+9.5 & 12 & 1965 field $B$ \\
\hline+24.4 & 3 & PLS field 81-82, averaged \\
\hline+29.8 & 3.5 & PLS field 91-92, averaged \\
\hline+35.0 & 1.5 & PLS field $101-102$, averaged \\
\hline
\end{tabular}

pretended to have a limiting magnitude of 20.5 , which now seems rather optimistic. The PLS numbers are here brought back to a limiting magnitude of 20.0 ; the unit area is the size of a survey plate, about $6.5 \times 6.5$.

The results of the 1965 survey have been adapted in a similar way, but one more correction was necessary here. Since in 1965 no orbits were obtained, the 1965 numbers contained an unknown number of Hilda-type asteroids. A frequency plot of the displacements of the supposed Trojans from the one blink plate to the other gave the impression that the Hildas could be separated rather easily; they clearly stood out as a secondary maximum. The number of Trojans from the quick survey has been diminished accordingly.

The nine Trojans to be added to the T-2 result were distributed as follows: to the number of Trojans found in each field 2 were added, and 1 more was assigned to the field having the lowest amount of Trojans. These numbers, together with the revised counts of 1960 and 1965, are given in Table VII. The distances of the four program fields to L4 are given in Table I; in the sign convention adopted in the 1970 paper (Van Houten et al. 1970a) these distances are given negative sign, because the T-2 fields are situated between L 4 and Jupiter. Figure 3 shows these results in graphical form; the line drawn through the points gives the apparent best value of the frequency function of L4 Trojans along the ecliptic and will be used in the determination, given below, of the L4/L5 Trojan number ratio. It is hoped that a more reliable value of this ratio can be given after the completion of the T-1 survey.

\section{THE $N($ L4)/N(L5) RATIO}

The only one of our survey programs on L5 that has been finished up to now is T-3. This gives only a single point in Fig. 3, where it is denoted by a cross. Comparison 


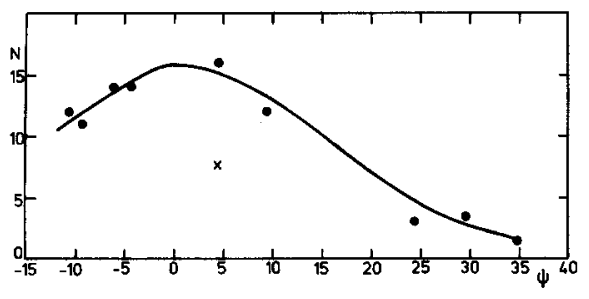

FIG. 3. The number of Trojans brighter than 20.0 mag. as a function of heliocentric distance from libration point $\mathrm{L} 4$ along the ecliptic; for a field of size $6.5 \times 6.5$. The single L5 result of 1977 is indicated by a cross.

with the L4 curve yields an L4/L5 ratio of 2.0. If the spread of the individual points around the L4 curve is also representative of the uncertainty in the L5 result, this number cannot be far from the truth. In that case the density of L4 Trojans in the ecliptic is 1.5 to 2.5 times that of the L5 Trojans.

This result does not necessarily pertain to the total L4/L5 ratio if the distribution of orbital inclinations is different for L4 and L5 Trojans. Whether this is so can be tested, however, because for both the T-2 and T-3 Trojans (and those found in the PLS) orbital elements are available. We corrected the numbers found for latitude-cutoff using the following expressions.

If the material is complete for inclinations smaller than $i_{0}$, those larger than this limit will be incomplete because of the latitude cutoff of the material; the fraction of such objects within the strip is

$$
\frac{2}{\pi} \arcsin \left(\frac{\sin i_{0}}{\sin i}\right) \quad \text { (Kiang 1971). }
$$

The value of $i_{0}$ can be computed from the expression

$$
5.2 \sin \left(b_{\mathrm{m}}-i_{0}\right)=\sin b_{\mathrm{m}},
$$

$b_{\mathrm{m}}$ being the maximum latitude of the field investigated.

This procedure is only valid if there are no longitudedependent irregularities in the density of Trojans. Figure 3 gives the impression that this distribution is smooth close to the ecliptic, and, since all high-inclination Trojans cross this zone during their orbital motion, the same can be inferred for the Trojans outside the region surveyed.

Figure 4 shows the distribution function of Trojan inclinations for L4 (mainly T-2 material, but with some PLS orbits) and L5 (only T-3 material). The numbers are averaged over inclination intervals of $5^{\circ}$. The dashed curve indicates the numbers corrected for latitude cutoff. The values derived for $i_{0}$ are 4.85 for the L4 fields and 12.14 for the L5 field, on account of the long strip observed in 1977. All Trojan orbits from our programs were used in these statistics, not only those of objects brighter than opposition magnitude 20.0 .

It is seen that the two distributions are different indeed; whereas there are numerous small inclination orbits around L4 they are mostly absent in L5.

The ratio corrected/observed number of Trojans, according to Fig. 4 , is 2.79 for the $L 4$ fields and 1.67 for the L5 strip of 1977; in this strip 25 Trojans were found brighter than 20.0 mag after correction for incompleteness. Applying these corrections to the results of Fig. 3 we obtain for the true spatial ratio of the numbers of Trojans around L4 and L5:

$$
N(\mathrm{~L} 4) / N(\mathrm{~L} 5)=30 \times 2.79 / 25 \times 1.67=2.0,
$$

the same as the ratio for the ecliptic plane. The reason for this is that, although the number of large-inclination Trojans is relatively larger around $\mathrm{L} 5$ than around $\mathrm{L4}$, in an absolute sense these numbers are still small, and therefore do not contribute much to the total number of Trojans. Since the main uncertainty of this number is caused by the T-3 result of 1977 , the accuracy of this ratio can again be estimated as being \pm 0.5 .

The ratio 2.0 was found earlier by Shoemaker $e t$ al. (1989), who also found that the ratio is unity if only bright Trojans are taken into account. This cannot be checked in the present investigation. The result of our surveys is compatible with the conclusions of Shoemaker et al.
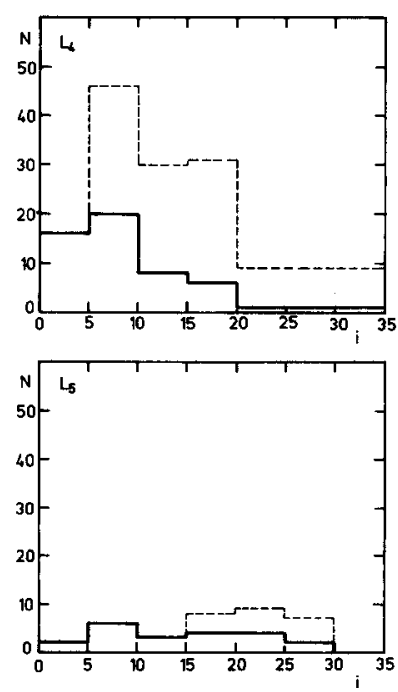

FIG. 4. Block diagram of the numbers of Trojans as a function of orbital inclination, counted in strips $5^{\circ}$ wide, for the surroundings of L4 and L5. Full-drawn lines: observed Trojans; dashed lines: numbers corrected for latitude cutoff. 
(1989) and the ratio derived here is valid only for faint Trojans (opposition magnitudes 19.0-20.0).

Degewij and Van Houten (1979) give 3.5 for this ratio, referring to a paper by Van Houten (1980, in preparation). This value was only preliminary, and was shown subsequently to be incorrect; therefore Van Houten (1980) was not published.

Integration of Fig. 3 yields the total number (96) of L4 Trojans brighter than 20.0 at opposition, within 6.5 from the ecliptic. Correction for the latitude cutoff brings this number to $536(=2 \times 2.79 \times 96$; the factor 2 is for integration above and below the ecliptic plane). Using the ratio between $N(\mathrm{~L} 4)$ and $N(\mathrm{~L} 5)$ derived above yields 268 for the number of L5 Trojans brighter than 20.0 in opposition. The total of the two libration points thus gives 804 Trojans above this limit. The L4 result is larger than that derived in the 1965 survey (Van Houten et al. 1970a), but smaller than the estimate of Shoemaker et al. (1989), which is about 800 for L4. This number was read from the curve marked "estimated population" in their Fig. 1, using $B(1,0)=13.82 \mathrm{mag}$ as equivalent for photographic magnitude 20.0 at opposition. This average value for $B(1,0)$ was determined experimentally from the PLS and the T-2 surveys, using $B(1,0)=g+0.10$.

The brightest Trojans have a different distribution of orbital inclinations; here the objects with $i>20^{\circ}$ make up $37 \%$ of the total, and there is no difference between L4 and L5 Trojans. This is different from the T-2 result derived above; it may be explained in two different ways: (a) the orbital inclination function of faint L4 Trojans is different from that of the bright ones, or (b) the high inclination objects in the T-2 survey were systematically missed during blinking, for whatever reason. There are some indications that the second explanation is the right one.

In order to obtain an orbital inclination distribution equal to that of the bright Trojans 4 to 5 objects with $i>$ $20^{\circ}$ have to be added to the material of Fig. 4. This results in the addition of 40 objects in the corrected distribution. The ratio: corrected/observed number of L4 Trojans is increased by this addition to 3.26 , the same factor as found by Shoemaker et al. (1989), who also used for its derivation the inclination distribution of the bright Trojans. The total number of L4 Trojans with opposition magnitude brighter than 20.0 is increased to 626 and the ratio $N(\mathrm{~L} 4) / N(\mathrm{~L} 5)$ becomes 2.3 .

The distribution curve of $\mathrm{L} 4$ Trojans given by Shoemaker et al. (1989) from which the number of $800 \mathrm{~L} 4$ Trojans with $m<20.0$ at opposition, as given above, was derived, was obtained by them by applying several corrections to the distribution function given in Van
Houten et al. (1970a). One of these corrections must be rejected: that for the PLS Trojans found after the publication of the 1970 paper (Van Houten et al. 1970b). Since the numbers used in Van Houten et al. (1970a) had been corrected for incompleteness no correction for Trojans found later is needed. Moreover the objects found in the October fields of the PLS were not used in these statistics. This decreases the Shoemaker et al . (1989) number for L4 Trojans with $m<20.0$ at opposition to 745 . A further possible cause of the difference with our value of 626 may be their transformation of photographic opposition magnitudes to $B(1,0)$ for the 1965 survey, where no orbits were available. How this was done is not mentioned by the authors.

\section{ACKNOWLEDGMENTS}

It is a pleasure to thank Palomar Observatory for its hospitality and for the observing time on the 122-cm Schmidt Telescope. Thanks are also due to the director of Kapteyn Laboratorium at Groningen and to Dr. W. W. Shane of the Catholic University at Nijmegen for making their plate measuring instruments available to us for this program. The many very useful remarks of Dr. E. Bowell are gratefully acknowledged.

\section{REFERENCES}

Bowell, E., T. Gehrels, and B. H. Zellner 1979. Magnitudes, colors, types and adopted diameters of the asteroids. In Asteroids (T. Gehrels, Ed.), p. 1108-1129, Univ. of Arizona Press, Tucson.

Degewis, J., AND C. J. VAN Houten 1979. Distant asteroids and outer Jovian satellites. In Asteroids (T. Gehrels, Ed.), pp. 417-435, Univ. of Arizona Press, Tucson.

Gehrels, T. 1956. Photometric studies of asteroids. V. The light-curve and phase function of 20 Massalia. Astrophys. J. 123, 331-338.

KIANG, T. 1971. The distribution of asteroids in the direction perpendicular to the ecliptic plane. In Physical Studies of Minor Planets. (T. Gehrels, Ed.), pp. 187-193. NASA, Washington, DC.

Shoemaker, E. M., C. S. Shoemaker, ANd R. F. Wolfe 1989. Trojan asteroids: Populations, dynamical structure and origin of the L4 and L5 swarms. In Asteroids II (R. P. Binzel, T. Gehrels, M. S. Matthews, Eds.), pp. 487-523. Univ. of Arizona Press, Tucson.

Stebins, J., A. E. Whitford, AND H. L. Johnson 1950. Photoelectric magnitudes and colors of stars in Selected Areas 57, 61 and 68. Astrophys. J. 112, 469-476.

Van Houten, C. J., I. Van Houten-Groeneveld, and T. Gehrels 1970a. The density of Trojans near the Preceding Lagrangian point. Astron. J. 75, 659-662.

Van Houten, C. J., I. Van Houten-Groeneveld, P. Herget, and T. GEHRELS 1970b. The Palomar-Leiden survey of faint minor planets. Astron. Astrophys. Suppl. 2, 339-448.

Van Houten-Groeneveld, I., C. J. Van Houten, M. WisseSchouten, C. Bardwell, and T. Gehrels 1989. The 1977 Palomar-Leiden Trojan survey. Astron. Astrophys. 224, 299-302. 